\title{
Prevalence of acute neurology: a 2-week snapshot in a district general hospital
}

\author{
Authors: Meneka Kanagaratnam, ${ }^{A}$ Ajay Boodhoo, ${ }^{B}$ Bridget $\mathrm{K} \mathrm{MacDonald}^{\mathrm{C}}$ and Arani Nitkunan ${ }^{\mathrm{C}}$
}

\begin{abstract}
Neurological illnesses are a common cause of hospital admissions, yet most patients are not primarily managed by neurologists. Acute neurology service provision varies across the UK. This study aimed to establish the number of neurological admissions during a 2-week period in a district general hospital, as well as the proportion of those patients who were seen by the hyperacute neurology team. This was done by analysing the diagnostic codes. It was established that there was a total of 2,242 admissions during the study period and, of those, 491 (22\%) had neurological codes. Analysis of the 491 hospital electronic records revealed that 229 (10\%) were truly neurologically relevant and, of those, $14 \%$ of patients received an opinion from the neurology team. Therefore, it can be concluded that only a small proportion of acute neurology is currently being seen by neurologists.
\end{abstract}

KEYWORDS: Acute neurology, neurologist, nurse specialist

DOI: 10.7861/clinmed.2019-0377

\section{Introduction}

In recent years, an ever increasing amount of healthcare data are being collected. This is not only vital in picking up early warnings of excess mortality and morbidity, but also allows healthcare performances such as hospital admission rates, length of stay and readmission rates to be compared across individual trusts. NHS RightCare (part of NHS England) is using this data in healthcare planning. ${ }^{1}$ The information is based exclusively on diagnostic codes provided by individual hospital informatics departments. These are used by providers and commissioners to determine the Healthcare Resource Group codes which are designed to group together conditions which use common levels of healthcare resource. ${ }^{2}$ It is on this basis that inpatient care is funded.

Although neurological illnesses are the third commonest cause of admission after cardiorespiratory conditions, until recently little attention has been paid to the way acute neurological patients are cared for. ${ }^{3}$ In 2010, hyperacute stroke units were established in

Authors: Afoundation year 2 doctor, St George's University Hospitals NHS Foundation Trust, London, UK; ${ }^{\text {Plead neurology }}$ nurse, Croydon University Hospital, Thornton Heath, UK;

${ }^{c}$ consultant neurologist, Croydon University Hospital, Croydon, UK
London, aiming to admit all suspected cases of stroke - of which about $25 \%$ are 'stroke mimics'. ${ }^{4}$ Detailed information on stroke care has been collected since 2013 in the Sentinel Stroke National Audit Programme. ${ }^{5}$ No such comparative data are available for the performance of different trusts' care of acute neurology.

In order to be able to assess all aspects of acute neurological care, it is essential that a comparative model is established similar to that in stroke medicine. An obvious way to do this seems to be to use the same methodology as RightCare. Previous estimates of the percentage of acute admissions with neurological diagnoses have suggested a figure of $10-20 \%$. However, these estimates have not been based on modern methods of diagnostic coding but physical reviewing of notes. ${ }^{3} \mathrm{~A}$ more recent paper by Chapman et al found that $9 \%$ of admissions were neurological. ${ }^{6}$

The first aim of this study was to determine the number of patients admitted to a single district general hospital (DGH) with primary and/or secondary neurological diagnoses. This was done with more widely used methodology based on diagnostic codes. Our second aim was to determine how much of the acute neurology being admitted was actually referred to and reviewed by neurologists, using the neurology department's comprehensive database.

\section{Methods}

Croydon University Hospital provides healthcare for a population of approximately 380,000 people. ${ }^{7}$ There are no inpatient neurology beds, thus a ward review service is provided by a team of consultant neurologists ( 3.2 whole time equivalents) and three nurse specialists, with no junior doctors. We identified all patients admitted to the acute medical unit (AMU), rapid AMU (RAMU) or inpatient wards at Croydon University Hospital between 06 and 19 March 2017, inclusive, using the database provided by the informatics department - this included emergency and elective patients. Patient demographics, admission date/time, location of admission, primary diagnoses and up to 12 secondary diagnoses were recorded. The diagnostic codes used were Systematized Nomenclature of Medicine Clinical Terms (SNOMED CT ${ }^{\circledR}$ ). We identified patients with a neurological primary diagnosis and separated them from those who had one in any of the other 12 diagnoses listed (referred to henceforth as a secondary diagnosis). For example, patients with transient loss of consciousness were usually coded as 'syncope and collapse'. These patients' notes were reviewed and included if there was any uncertainty or the cause was neurological (ie a seizure). Due to practical constraints, a false negative check could not be done, thus admissions without a neurological code were not analysed. 
Patients identified with a neurological problem had their diagnostic codes verified using their electronic notes. Patients were excluded from further analysis if review of notes revealed that there was a background of a neurological diagnosis which was not relevant to the presenting complaint (eg a patient with multiple sclerosis who had a hernia repair) and if their admission was to the day surgery unit / endoscopy / imaging department for a procedure/investigation. The remaining exclusion criteria are included in Table 1.

We then used the neurology department's database of all referrals made to neurology for the same period to determine which patients had been referred to and reviewed by the neurologists, as well as determine the number of patients, if any, who appeared on the neurology department database but not on the hospital database provided by the informatics department. Of note, patients who were reviewed by neurology on the ambulatory emergency care unit were included in the department's database of referrals but would not have appeared on the database provided by the informatics department, as such patients were not considered as being 'admitted'. The number of patients seen on this pathway may be an indicator of admission avoidance.

\section{Results}

A total of 2,242 patients were admitted during the study period, of whom 491 (22\%) were coded as having neurological diagnoses. On review, 262 patients (53\%) were excluded from the 491 identified, leaving 229 patients with a neurological diagnosis - either primary, secondary or both. Exclusions were made where the neurological diagnosis was incidental and not relevant to their admission, as shown in Table 1. Neurology patients therefore accounted for $10 \%$ (229) of all admissions ( $n=2,242)$. Only $23(10 \%)$ of 229 had been referred to and reviewed by a neurologist (Fig 1).

All the patients referred to neurology were reviewed, with the exception of one patient who was discharged prior to review (this one patient has been counted as a 'seen' in an intention to treat manner). Two were not referred to neurology but were discussed informally. A further six patients who were not referred were discussed with the local tertiary centre, St George's Hospital (five with neurosurgery and one with stroke).
If these eight patients are included, 31 (14\%) patients with a primary or secondary neurological diagnosis were assessed by a neurologist or a neurosurgeon. Ninety-three patients had primary diagnostic codings, and only 17 (18\%) of those were reviewed by a neurologist. For the 136 patients with solely secondary neurological codes, six (4\%) were reviewed (Fig 1).

\section{Neurological diagnoses}

The commonest three neurological codes for the 93 patients with a primary neurological diagnosis were headaches (19\%) followed by syncope (17\%) and epilepsy (13\%). Table 2 lists the commonest diagnostic codes for those with a primary and secondary neurological diagnosis. Of note, there is a wide spread of other diagnoses which have been grouped together into 'other', including 'other and unspecified convulsions' (ie non-epileptic attacks), intracranial abscesses and 'other and unspecified symptoms/signs involving general sensations/perception' (ie functional neurological disorders).

\section{Comparison with the neurology department's database of referrals}

The neurology department's database showed that 56 patients were reviewed during the study period. Only 36 of those patients $(64 \%)$ were identifiable on the database provided by informatics with the correct neurological diagnostic coding. Of the remaining 20 patients, three had no neurological diagnosis at all coded but were on the departmental database, 10 did not appear on the database provided by informatics due to their admission date being before the study period, five had been reviewed in the ambulatory unit (and not in the RAMU, AMU or inpatient wards; therefore were not considered as inpatients) and two had missing data (Fig 2).

Examples of incorrect coding.

> A patient had presented with urinary retention and had relapsing-remitting multiple sclerosis, but the coding was 'urinary tract infection' only.

> A patient was seen by neurology due to speech disturbance, but the primary diagnosis was precordial pain.

$>$ A patient was seen due to possible meningitis but this was coded as sepsis (as sepsis is not a neurological code, the patient was excluded at the first stage)

Table 1. A table showing the exclusion criteria for this study, and the number of patients excluded

\section{Reason for exclusion}

Neurological diagnosis as a past medical history not relevant to presenting complaint

Admitted to the day surgery unit / endoscopy / imaging department for a procedure/investigation

Alcohol withdrawal/intoxication

Mechanical falls / injury / uncomplicated low back pain

Delirium

Uncomplicated head injury / faint

Stroke (seen by the stroke team)

Incorrect coding

Total

\section{Number of patients} 64 


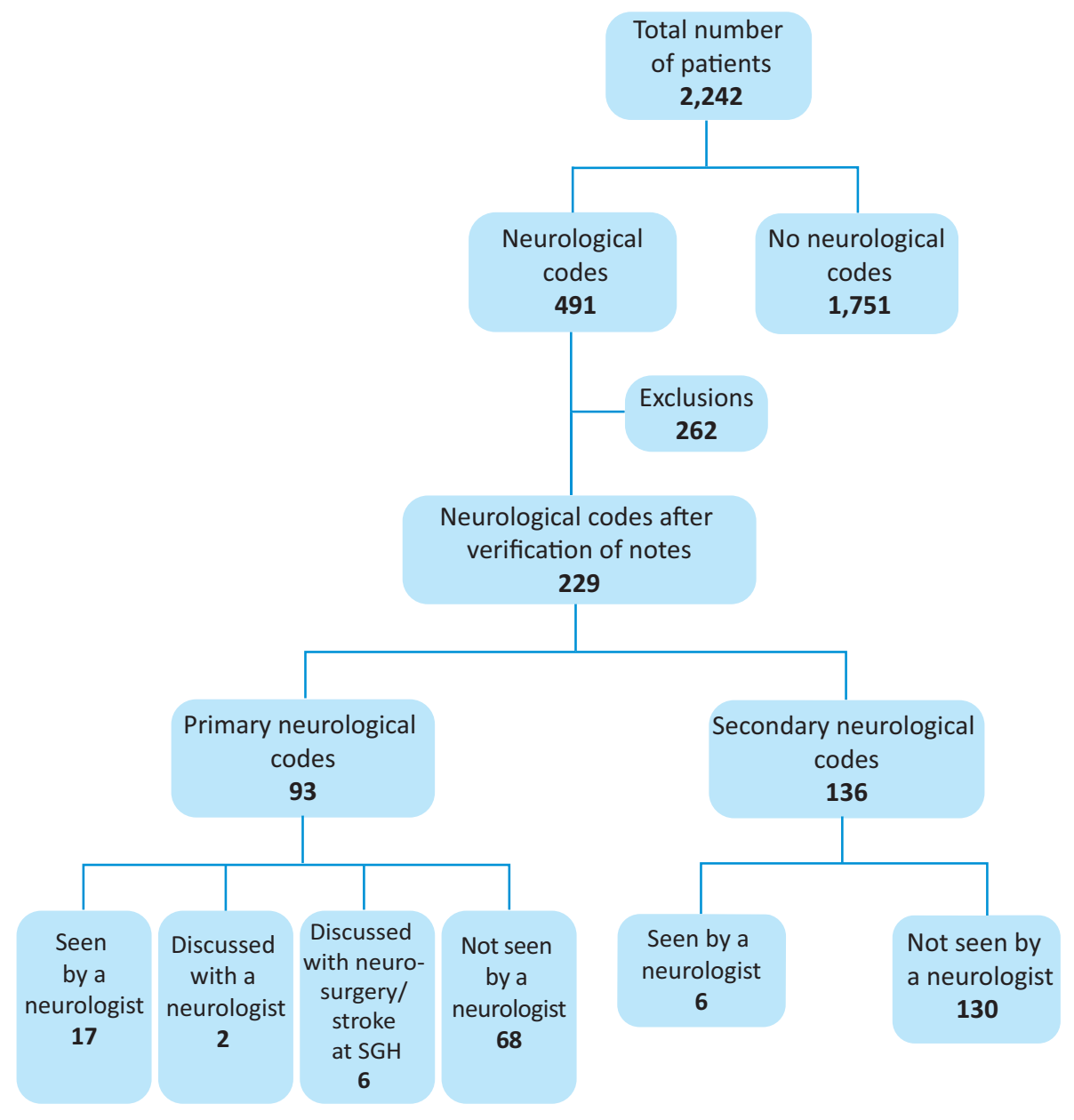

Fig 1. A flowchart showing the number of patients presenting with neurological diagnoses, and who were referred to and seen by neurology. SGH = St George's Hospital.

\section{Discussion}

This paper raises serious, but not new, concerns regarding national data collection methods. ${ }^{8}$ We have concentrated specifically on neurological admissions to a single DGH. The hospital codings showed that, of the 2,242 admissions during the study period, 491 had neurological codes. By looking up each of those 491 cases on their individual hospital electronic records, it was clear only 229 were truly neurologically relevant. We accept that this figure may also be inaccurate because we were not able to check all 2,242 cases.

Our first question was 'What percentage of admissions were neurological in nature?' Our study suggests that 10\% of admissions had a neurological diagnosis, with only $4 \%$ having a primary neurological diagnosis. This is comparable to Chapman et al's figure of $9 \%$. However, that study included stroke. ${ }^{6}$ We believe that our study is unique in its use of methods based on diagnostic codes, a concept used systematically by NHS England. Three of the 57 patients (5\%) reviewed by neurology were incorrectly coded. This highlights a significant limitation of this study and may be an underrepresentation of the well-recognised problem of coding.

Our second question was 'How much of the acute neurology do we actually see?'

As we run a very comprehensive service from $9 \mathrm{am}-5 \mathrm{pm}$ and 5 days per week, we had assumed that we saw the bulk of the acute neurology. ${ }^{9}$ This study, however, suggests that of the 229 relevant patients coded as having a primary or secondary neurological condition, only $14 \%$ (31) were referred to and reviewed. Seventeen (18\%) of the 93 patients with a primary neurological code were seen. The review of the diagnostic codes of the patients that were reviewed mirrored the codes seen overall and therefore shows that there is no particular theme or reason which prompts a neurological referral. This data implies that we are simply being referred a fraction of the patients along the spread of diagnoses. Although we did not collect specific data on whether the patient was referred because of the presentation or whether it was opportunistic, one could wrongly assume that a referral on a patient with a neurological primary diagnosis was due to the presentation and a secondary diagnosis was opportunistic, as sometimes the two are intrinsically linked (eg confusion secondary to an urinary tract infection or infection affecting the mobility of a patient with Parkinson's disease). Almost three-quarters of the patients reviewed by neurology (17/23) had a primary neurological diagnosis. Therefore, we could extrapolate that the majority were referred because of the presenting problem and it is difficult to comment on the rest as they may be indeed linked.

Another key issue of how much we see is linked to when we see them. Ten patients were seen during this study period who were 


\begin{tabular}{|c|c|}
\hline Neurological codes & Frequency, \% (n) \\
\hline \multicolumn{2}{|l|}{ Primary } \\
\hline Headache & $19.4(18)$ \\
\hline Syncope & $17.2(16)$ \\
\hline Epilepsy & $12.9(12)$ \\
\hline Head injury & $7.5(7)$ \\
\hline Parkinson's disease & $6.5(6)$ \\
\hline $\begin{array}{l}\text { Signs / symptoms involving cognition / } \\
\text { awareness }\end{array}$ & $5.4(5)$ \\
\hline Neoplasm & $3.2(3)$ \\
\hline Radiculopathy & $3.2(3)$ \\
\hline Disorientation & $2.2(2)$ \\
\hline Dementia & $2.2(2)$ \\
\hline Other & $20.4(19)$ \\
\hline Total & $100(93)$ \\
\hline \multicolumn{2}{|l|}{ Secondary } \\
\hline Dementia & $22.8(31)$ \\
\hline Epilepsy & $11.8(16)$ \\
\hline Disorientation & $8.8(12)$ \\
\hline Parkinson's disease & $8.1(11)$ \\
\hline Headache & $7.4(10)$ \\
\hline Cerebrovascular disease & $6.6(9)$ \\
\hline Syncope & $4.4(6)$ \\
\hline Multiple sclerosis & $2.2(3)$ \\
\hline Cerebral palsy & $2.2(3)$ \\
\hline Other & $25.7(35)$ \\
\hline Total & $100(136)$ \\
\hline
\end{tabular}

not admitted during the 2-week period. We have not delved into the reason underlying when referrals were made. However, we are finding that since computerisation of the referral system and with our nurse specialists acting as coordinators, our referrals are increasing and we are seeing more patients on the acute unit. ${ }^{9}$ Ten patients were seen in the ambulatory unit: five of whom were not admitted, and could be an indication of patients whose admission can be avoided with prompt review; five required admission, one for deterioration of her primary progressive multiple sclerosis and two for investigation of their headache (followed by prompt investigation and discharge), another was electively readmitted for a day for temporal artery biopsy for possible giant cell arteritis the day after she was reviewed while one whose cerebral toxoplasmosis did not require admission but other respiratory complications of HIV did. We are hoping to expand this ambulatory service but will have to ensure that there is careful gating of this service to ensure that it does not become a service of reassurance.
This leads to a third key question 'How much of the acute neurology do neurologists need to see?' Clearly there will be widely differing opinions on this critical issue. There is minimal evidence to support our view - perhaps the best comparative models are hyperacute stroke units, transient ischaemic attack (TIA) clinics and first fit clinics. All these three models run on the basis that in order to see all patients with the relevant primary diagnoses namely stroke, TIAs and epileptic seizures, it is necessary to see far more patients than have the final positive diagnosis because of so-called 'mimics'. ${ }^{4}$ We would echo the opinion of Warlow et al that, if adequately funded/supported, neurologists are best placed to provide an acute neurology service. ${ }^{10}$ Getting It Right First Time have proposed a categorisation of when patients with particular conditions should be under the care of a neurologist, and this could be used to determine when a patient, where there are not neurology inpatient beds, should at least be reviewed; for example, encephalitis / epilepsy / multiple sclerosis / myasthenia are categorised as 'definitely' while meningitis / cranial nerve disorders / headaches / motor neurone disease / Parkinson's disease are categorised as 'probably'. ${ }^{11}$ However, when the diagnosis is not known, this is not so useful.

Reviewing only $18 \%$ of patients admitted with a primary neurological code, despite the existence of a 9am-5pm 5-day service, implies the need to shift away from scheduled care to unscheduled care, as proposed by the Association of British Neurologists in $2011 .^{12}$ This requires a significant overhaul in the way acute neurological care is structured and commissioned. This will of course have financial implications for trusts, as currently outpatient work demands a tariff whereas inpatient work (ward review) does not (although work by Boodhoo et al and Moodley et al show that there is a potential impact on length of stay and admission avoidance which would be of, not only clinical but also, financial benefit). ${ }^{9,13}$

Our experience from seeing significant numbers of ward referrals is that it is only obvious in hindsight which referrals were unnecessary. Experience shows that a serious sounding monoplegia can have a painful joint and the most innocuous headache could be cryptococcal meningitis. Referrals are being made, at times, by quite junior medical staff. We reiterate here from our earlier paper the Donald Rumsfeld quote 'unknown unknowns.' ${ }^{9}$ This is why we feel that a specialist should see as many as is realistic.

\section{Conclusion}

Our paper raises concerns about the accuracy of current data collection methods. Secondly it suggests that about $10 \%$ of admissions (excluding stroke) are due to a neurological problem, which is in keeping with other studies and thirdly, that less than a quarter of acute neurology is being seen by neurologists, even in a trust with a comprehensive 5 -day service in place.

\section{Acknowledgements}

We would like to thank Dr Fred Schon for his very helpful contribution and to the rest of the neurological team at Croydon for enabling us to provide this service (Francesca Mastrolilli, Hamid Modarres, Medina Southam and Glenda Lastrilla). 


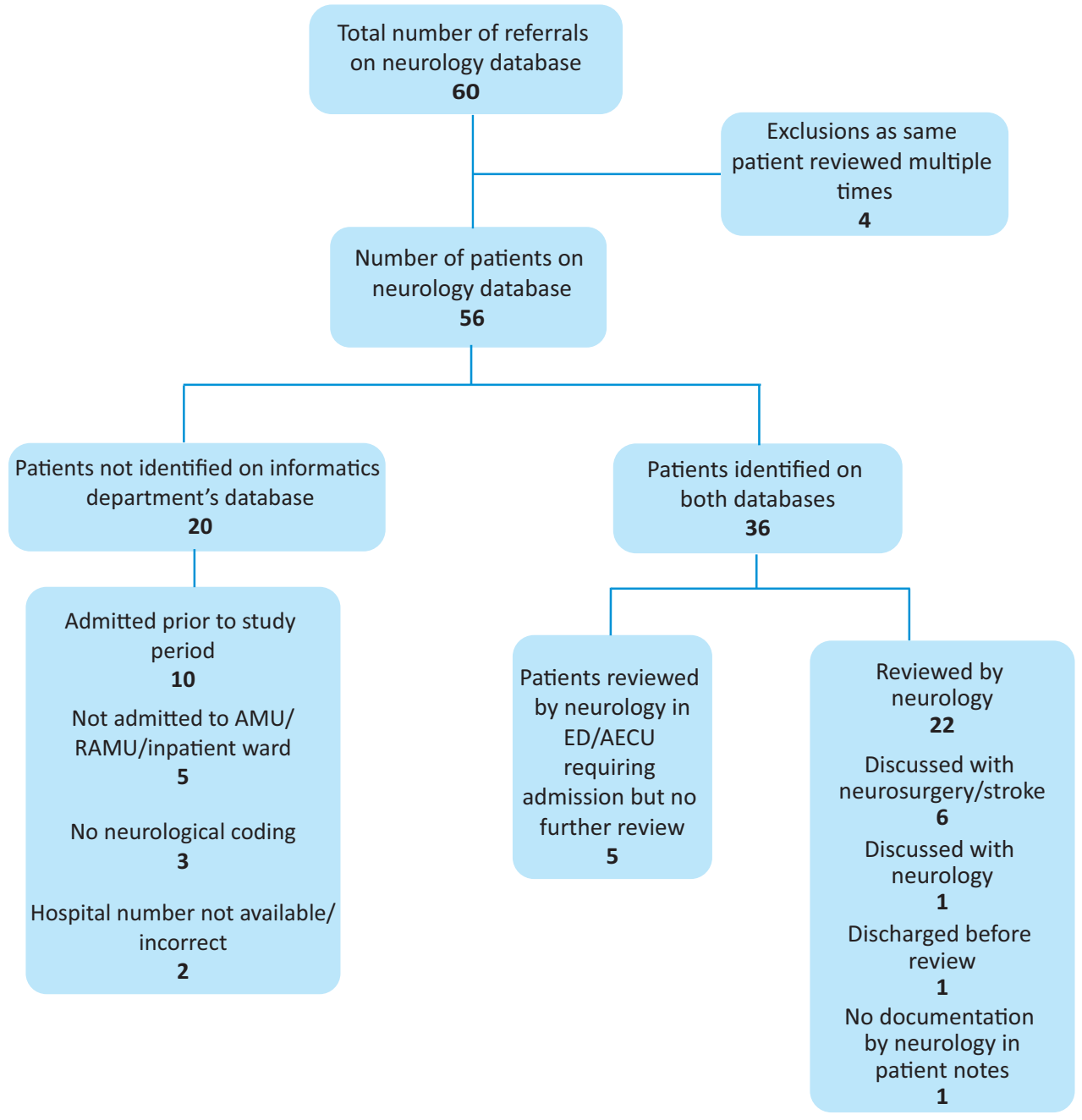

Fig 2. A flowchart showing the issues identified with the patients listed on the neurology departmental database as compared to the informatics departmental database. AECU $=$ ambulatory emergency care unit; $\mathrm{AMU}=$ acute medical unit; $\mathrm{ED}=$ emergency department: RAMU = rapid acute medical unit.

\section{References}

1 NHS England. What is NHS RightCare? NHS. www.england.nhs.uk/ rightcare/what-is-nhs-rightcare [Accessed 16 September 2018].

2 NHS. Healthcare resource groups. NHS. www.datadictionary.nhs.uk/ data_dictionary/nhs_business_definitions/h/healthcare_resource_ group_de.asp [Accessed 13 April 2019].

3 Weatherall MW. Acute neurology in a twenty-first century district general hospital. J R Coll Physicians Edinb 2006;36:196-200.

4 Dawson A, Geoffrey GC, Pereira AC et al. Stroke mimic diagnoses presenting to a hyperacute stroke unit. Clin Med 2016;16:423-6.

5 Sentinel Stroke National Audit Programme. About SSNAP. King's College London. www.strokeaudit.org/About-SSNAP.aspx [Accessed 16 September 2018]

6 Chapman FA, Pope AE, Sorensen D, Knight RSG, Al-Shahi Salman R. Acute neurological problems: frequency, consultation patterns and the uses of a rapid access neurology clinic. J R Coll Physicians Edinb 2009;39:296-300.

7 Croydon Health Services NHS Trust. About us: Who we are and what we do. NHS. www.croydonhealthservices.nhs.uk/about-us [Accessed 16 September 2018].

8 Arksey H, O'Malley L. Scoping studies: towards a methodological framework. Int ] Soc Res Methodol 2005;8:19-32.

9 Nitkunan A, MacDonald BK, Boodhoo A et al. A hyperacute neurology team - transforming emergency neurological care. Clin Med 2017:17:298-302

10 Warlow C, Humphrey P, Venables G. UK neurologists and the care of adults with acute neurological problems. Clin Med 2002;2:436-9.

11 Fuller G, Connolly M, Mummery C, Williams A. GIRFT neurology methodology and initial summary of regional data. NHS, 2019. https://gettingitrightfirsttime.co.uk/wp-content/uploads/2017/07/ GIRFT-neurology-methodology-090919-FINAL.pdf [Accessed 18 November 2019].

12 Royal College of Physicians. Local adult neurology services for the next decade. Report of a working party. London: RCP, 2011.

13 Moodley K, Jones V, Yogarajah M et al. Hyperacute neurology at a regional neurosciences centre: a 1-year experience of an innovative service model. Clin Med 2019;19:119-26.Authors: Meneka

Address for correspondence: Dr Arani Nitkunan, Neurology Department, Croydon University Hospital, 530 London Road, Croydon CR7 7YE, UK.

Email: anitkunan@nhs.net 